\title{
Evaluating The Performance of Several Data Preprocessing Methods Based On GRU in Forecasting Monthly Runoff Time Series
}

\section{Wenchuan Wang ( $\nabla$ wangwen1621@163.com )}

North China University of Water Resources and Electric Power https://orcid.org/0000-0003-1367-5886

\section{Yu-jin Du}

North China University of Water Resources and Electric Power

Kwok-wing Chau

The Hong Kong Polytechnic University

Chun-Tian Cheng

Dalian University of Technology

\section{Dong-mei Xu}

North China University of Water Resources and Electric Power

\section{Wen-Tao Zhuang}

North China University of Water Resources and Electric Power

\section{Research Article}

Keywords: monthly runoff prediction, data preprocessing techniques, gated recurrent unit network, variational mode decomposition, wavelet packet decomposition

Posted Date: November 3rd, 2021

DOl: https://doi.org/10.21203/rs.3.rs-868259/v1

License: (c) (i) This work is licensed under a Creative Commons Attribution 4.0 International License.

Read Full License 


\section{Evaluating the performance of several data preprocessing methods based on GRU in forecasting monthly runoff time series}

\section{Wen-chuan Wang}

College of Water Resources, Henan Key Laboratory of Water Resources Conservation and Intensive Utilization in the Yellow River Basin, North China University of Water Resources and Electric Power, Zhengzhou, 450046, People's Republic of China

Corresponding author, E-mail: wangwen1621@163.com; wangwenchuan@ncwu.edu.cn

\section{Yu-jin Du}

College of Water Resources, Henan Key Laboratory of Water Resources Conservation and Intensive Utilization in the Yellow River Basin, North China University of Water Resources and Electric Power, Zhengzhou, 450046, People's Republic of China E-mail:2366036835@qq.com

\section{Kwok-wing Chau}

Department of Civil and Environmental Engineering, The Hong Kong Polytechnic University, Hung Hom, Kowloon, Hong Kong, People's Republic of China

E-mail: cekwchau@polyu.edu.hk

\section{Chun-Tian Cheng}

Institute of hydropower system \& Hydroinformatics, Dalian University of Technology, Dalian, 116024, People's Republic of China

E-mail: ctcheng@dlut.edu.cn

\section{Dong-mei Xu}

College of Water Resources, Henan Key Laboratory of Water Resources Conservation and Intensive Utilization in the Yellow River Basin, North China University of Water Resources and Electric Power, Zhengzhou, 450046, People's Republic of China E-mail: xudongmei@ncwu.edu.cn

\section{Wen-Tao Zhuang}

College of Water Resources, Henan Key Laboratory of Water Resources Conservation and Intensive Utilization in the Yellow River Basin, North China University of Water Resources and Electric Power, Zhengzhou, 450046, People's Republic of China E-mail: 1026151299@qq.com 
Abstract: The optimal planning and management of modern water resources depends highly on reliable and accurate runoff forecasting. Data preprocessing technology can provide new possibilities for improving the accuracy of runoff forecasting, when basic physical relationships cannot be captured using a single prediction model. Yet, few researches evaluated the performances of various data preprocessing technology in predicting monthly runoff time series so far. In order to fill this research gap, this paper investigates the potential of five data preprocessing techniques based on gated recurrent unit network (GRU) model in monthly runoff prediction, namely variational mode decomposition (VMD), wavelet packet decomposition (WPD), complete ensemble empirical mode decomposition with adaptive noise (CEEMDAN), extreme-point symmetric mode decomposition (ESMD), and singular spectrum analysis (SSA). In this study, the original monthly runoff data is first decomposed into a set of subcomponents using five data preprocessing methods; second, each component is predicted by developing an appropriate GRU model; finally, the forecasting results of different twostage hybrid models are obtained by aggregating of forecast results of the corresponding subcomponents. Four performance metrics are employed as the evaluation benchmarks. The experimental results from two hydropower stations in China show that five data preprocessing techniques can attain satisfying prediction results, while VMD and WPD methods can yield better performance than CEEMDAN, ESMD and SSA in both training and testing periods in terms of four indexes. Indeed, it is significantly important to carefully determine an appropriate data preprocessing method according to the actual characteristics of the study area. 
Keywords: monthly runoff prediction; data preprocessing techniques; gated recurrent unit network; variational mode decomposition; wavelet packet decomposition.

\section{Introduction}

Natural streamflow is the main source of water resources and plays an important role in agriculture, ecological environment and social economy (Meshram et al. 2020). Due to the increasingly serious water problem in recent years, the optimum utilization and distribution of water resources have attracted considerable attentions of hydrologists (Niu et al. 2020). Accurate and stable prediction of runoff is an important factor in decision making regarding efficient planning and operation of reservoirs, hydro-power generation, sediment transport in rivers, scheduling reservoirs releases, irrigation management decisions and other hydrological applications (Turner et al. 2020; Yaseen et al. 2016; Zuo et al. 2020). Due to the influence of precipitation, underlying surface conditions, evapotranspiration and other factors, runoff series yield instability, time-varying and randomness, so making it difficult to accurately predict runoff. In order to remedy this problem, many models, such as physically-based models and databased models, have been developed and reported in the relevant literature (Chen et al. 2020a; Chen et al. 2020b; Yue et al. 2020).

Physically-based models can reflect and simulate physical processes of the hydrological cycle. However, process-based models require multisource information, and the computation parameters need be optimized. However, many studies (Fragkiadaki et al. 2015; Ömer Faruk 2010) reported that the performance of data-based models were comparable to physically-based models, and they could work very well 
with limited data. They had better modeling speed without modeling the internal structure, and could be more efficient with the support of software, hardware and algorithm parallelization (LeCun et al. 2015). Artificial neural network (ANN) (Reddy et al. 2021), model trees (Ali et al. 2020), fussy (rule-based) systems (Yaseen et al. 2019), and support vector machine (SVM) (Bhandari et al. 2019) were the most commonly used data-based methods in hydrological time series forecasting.

However, due to the limitation of algorithm or computation, the number of hidden layers in earlier ANN model is limited. In recent years, this shortage has been solved with sensor networks, accelerated GPU computing, and extensive data collection (Xiang et al. 2020). To process input series, recurrent neural networks (RNNs), which contained GRU and long short-term memory (LSTM), were proposed and extensively used in many fields (Yu et al. 2018; Zhang and Liu 2020). LSTM was capable of overcoming the problem of learning long-term dependence in hydrological basin associated with the conventional $\mathrm{RNN}$, which was a promising alternative for hydrological processes (Kratzert et al. 2018). GRU was a variant of RNN, which maintained useful information and discarded useless information in dynamic sequence data, with benefits of retaining the advantages of RNN (Ravanelli et al. 2018). In recent years, researchers applied GRU to water level prediction (Pan et al. 2020), soil moisture modeling (Yu et al. 2021), energy futures price prediction (Wang and Wang 2021), and streamflow forecasting (Zhao et al. 2021). These studies indicated that GRU performed better than other artificial intelligence method in mid- to long-term time series forecasting. However, few studies investigated the performance of GRU in monthly 
runoff prediction, which needed further exploration.

Since runoff time series is nonstationary and nonlinear, single model always fails to attain a satisfactory performance. According to literature, hybrid models that combined network optimization (Feng et al. 2020) or data processing (Wang et al. 2015b) have recently attracted more and more attention of scholars. Among them, decomposition-based hybrid methods have been proposed widely. The most widely used decomposition technologies can be classified into four main categories: empirical mode decomposition (EMD)-based methods, wavelet-based methods, VMD, and SSA (Kisi and Cimen 2011; Wang et al. 2015a; Wang et al. 2013; Zhang et al. 2011; Zuo et al. 2020).

EMD, proposed by Huang et al. (1998), is able to adaptively decompose the input signal into several intrinsic mode functions (IMF) and a residue (Res). Many researchers have investigated the performance of EMD-based hybrid models by combining EMD with different prediction models. Tang et al. (2020) proposed a hybrid prediction method by combining multiple model fusion and EMD for chaotic time series. There were some insufficiencies in the application of EMD, such as mode mixing. To remedy this, some improved versions of EMD were proposed, such as ensemble EMD (EEMD), ESMD, complementary ensemble EMD (CEEMD), and CEEMDAN. Ren et al. (2015) comprehensively investigated the performance of EMDbased hybrid wind speed prediction models, and the results demonstrated that CEEMDAN-based hybrid models always performed the best. Hence, this paper focuses on the performance of extended EMD hybrid models in monthly runoff forecast. 
Wavelet-based methods are very effective tools for signal analysis in both frequency and time domains. Discrete wavelet transform (DWT) and WPD were the most widely used wavelet-based methods in mid- to long -term time series prediction. Liu et al. (2018) developed a hybrid wind speed forecasting model based on WPD, LSTM, and CNN. Seo et al. (2016) developed and investigated several hybrid models for daily river stage forecasting by combining WPD and three machine learning models. Although WPD had many advantages in processing data, it should be noted that WPD had the weakness of non-adaptability. As an entirely non-recursive tool, VMD was executed to extract the nonlinear harmonics and trends from changeable signal (He et al. 2019). Compared to EMD, VMD could avoid the mode mixing problem, in which one subsignal was decomposed into several subcomponents with clear differences (Duan et al. 2021). Owing to its convenience and efficiency, VMD was adopted as one of data preprocessing tools for monthly runoff forecasting. As a non-parametric algorithm, SSA was capable of adapting itself to the dataset without relying on the prior model (Elsner 2002). SSA had strict mathematical theory and it could accurately extract periodic formation, trend formation, and quasi periodic information of the signal (Liu et al. 2019). Therefore, SSA had great vitality in the field of time series prediction.

Due to the excellent performance in hydrological forecasting, the above data preprocessing technologies combined with GRU model are selected for runoff forecasting. However, despite numerous literatures on the performance of data preprocessing technologies, few literatures quantitatively compare the performance of these techniques on monthly runoff forecasting. In order to fill this research gap, this 
paper investigates the performance of five data preprocessing techniques based on GRU model in monthly runoff series prediction, namely VMD, WPD, CEEMDAN, ESMD, and SSA. Four performance metrics are employed as the evaluation benchmarks. The results indicate that VMD, and WPD algorithm can yield better performance than CEEMDAN, ESMD and SSA in both training and testing periods in practice.

The remainder of this paper is organized as follows. Section 2 introduces the related methodologies. The experimental results and discussions are shown in Section 3, and Section 4 concludes the paper.

\section{Methodologies}

\subsection{Gated Recurrent Unit (GRU)}

The GRU network proposed by Cho, et al. (2014) solved the problem of gradient exploding/vanishing in RNN, which had outstanding advantages of high precision and fast training (Greff et al. 2017). GRU is developed based on LSTM, but with the reduction of computational complexity of the model by improving the operation of gates in LSTM. The difference is that LSTM contains three function gates, while GRU only has two gated units, namely update and reset gates. The update gate determines the degree to which the information from the previous state will be passed to the future, and the reset helps to define what information should be ignored from the previous state. A typical structure of GRU is shown in Fig. 1. The specific expressions are as follows:

$$
\begin{aligned}
& z_{t}=\sigma\left(w_{z} \cdot\left[h_{t-1}, x_{t}\right]\right) \\
& r_{t}=\sigma\left(w_{r} \cdot\left[h_{t-1}, x_{t}\right]\right) \\
& \bar{h}_{t}=\tanh \left(w \cdot\left[r_{t} \cdot h_{t-1}, x_{t}\right]\right)
\end{aligned}
$$


where $z_{t}$ is the output of the update gate, $\sigma$ is the sigmoid function, $w_{z}$ is the

175 weight of the update gate, $x_{t}$ is the input of this hidden layer, $h_{t-1}$ is the output of

176 the last hidden layer, $r_{t}$ is the output of the reset gate, $w_{r}$ is the weight of reset gate,

$177 \overline{h_{t}}$ is the current memory content, $\tanh (\cdot)$ is tanh activation function, $h_{t}$ is final 178 memory at the current step.

Insert Fig. 1.

180

\subsection{Variational Mode Decomposition (VMD)}

As a new multi-resolution non-recursive variational method proposed by

Comparing with EMD, VMD avoids error caused in the process of recursive computation and ending effect. The theory of VMD is given as follows:

$$
\begin{gathered}
\min _{\left\{y_{k}\right\}\left\{\omega_{k}\right\}}=\left\{\left\|\sum_{k=1}^{K} \partial_{t}\left[\left(\delta(t)+\frac{j}{\pi t}\right) \otimes y_{k}(t)\right] e^{-j \omega_{k} t}\right\|_{2}^{2}\right\} \\
\text { s.t. } \sum_{k=1}^{K} y_{k}=f(t)
\end{gathered}
$$

where $f(t)$ is the input signal, $\mathrm{K}$ is the total number of the modes, $\mathrm{t}$ is the time script, $y_{k}$ is the kth mode, $w_{k}$ is the center frequency, $\delta$ is the Dirac distribution, $\otimes$ is the convolution operator. multipliers and penalty term are adopted, which is written as: 


$$
L\left(y_{k}, w_{k}, \lambda\right)=\alpha \sum_{k=1}^{K}\left\|\partial_{t}\left[\left(\delta(t)+\frac{j}{\pi t}\right) \otimes y_{k}(t)\right] e^{-j \omega_{k} t}\right\|_{2}^{2}+
$$

$$
\left\|f(t)-\sum_{k=1}^{K} y_{k}(t)\right\|_{2}^{2}+\left(\lambda(t), f(t)-\sum_{k=1}^{K} \mathrm{y}_{k}(t)\right)
$$

195 where $\lambda$ and $\alpha$ are Lagrange multiplier and penalty, respectively.

The augmented Lagrangain L is computed by Eq. (7), and the saddle point of $\mathrm{L}$ can be found by the alternate direction method for multipliers (ADMM). Based on the ADMM optimization method, $y_{k}, w_{k}$ and $\lambda_{k}$ are updated in two directions. The expression of $y_{k}, w_{k}$ and $\lambda_{k}$ are as follows:

$$
\left\{\begin{array}{c}
\hat{y}_{k}^{n+1}=\frac{\hat{f}(w)-\sum_{i \neq k} \hat{y}_{i}(w)+0.5 \hat{\lambda}(w)}{1+2 \alpha\left(w-w_{k}\right)^{2}} \\
w_{k}^{n+1}=\frac{\int_{o}^{\infty} w\left|\hat{\mathrm{y}}_{k}^{n+1}(w)\right|^{2} d w}{\int_{0}^{\infty}\left|\hat{\mathrm{y}}_{k}^{n+1}(w)\right|^{2} d w} \\
\hat{\lambda}^{n+1}(w)=\hat{\lambda}^{n}(w)+\tau\left(\hat{f}(w)-\sum_{i \neq k} \hat{\mathrm{y}}_{k}^{n+1}(w)\right)
\end{array}\right.
$$

where $\hat{f}(w), \hat{y}_{i}(w), \hat{\lambda}(w)$, and $\hat{y}_{k}^{n+1}$ are the Fourier transform of $f(t), y_{i}(t)$, $\lambda(t)$, and $\hat{u}_{k}^{n+1}(t)$, respectively, $n$ is the iteration number, $\tau$ is the time step of dual ascent.

\subsection{Wavelet Packet Decomposition (WPD)}

WPD is an efficient signal preprocessing technology, which can split the original signal into approximation coefficients and detail coefficients. The wavelet basis function and decomposition levels can significantly influence the performance of WPD.

WPD contains DWT and CWT. CWT is shown as follows:

$$
C W T_{\mathrm{x}}(a, b)=\left\langle x(t), \psi_{a, b}(t)\right\rangle=\int x(t) \psi^{*}((t-b) / a) / \sqrt{a} d t
$$


where $x(t)$ is the input, $*$ is a complex conjugate, $b$ is a translation parameter, $a$ is a scale parameter, $b$ is a translation parameter, $\psi(t)$ is the mother wavelet function. $a$ and $b$ in DWT are:

$$
\left\{\begin{array}{c}
a=2^{i} \\
b=j 2^{i}
\end{array}\right.
$$

214 where $i$ and $j$ denote scale and translation parameters, respectively.

\subsection{Complete Ensemble Empirical Mode Decomposition with Adaptive Noise}

\section{(CEEMDAN)}

CEEMDAN is an improvement of EMD and EEMD. White Gaussian noise with certain standard deviation is employed to avoid the mode mixing problem. CEEMDAN greatly reduces the computational complexity by adding limited adaptive white noise in each decomposition. Meanwhile, owing to its decomposition and complete reconstruction, CEEMDAN is able to solve the mode mixing problem. The processes of CEEMDAN are as follows:

(1) White Gaussian noise series $\omega^{i}(n)$ is added to the original signal $s(n)$ and the obtained signal can be expressed as:

$$
s^{i}(t)=s(t)+\varepsilon_{0} \omega^{i}(t)
$$

where $i$ denotes the number of trials.

(2) The first IMF is obtained by EMD as follows:

$$
I M F_{1}(t)=\left(\sum_{i=1} I M F_{1}^{I}(n)\right) / I
$$

(3) Compute the first Res $r_{1}(t)$ by:

$$
r_{1}(t)=s(t)-I M F_{1}(t)
$$


(4) Compute the kth Res as:

$$
r_{k}(t)=s(t)-I M F_{k}(t)
$$

(5) Decompose the noise-added Res to attain the $(\mathrm{k}+1)$ th IMF by:

$$
I M F_{\mathrm{k}+1}(t)=\left(\sum_{i=1}^{I} E_{1}\left(r_{k}(t)+\varepsilon_{k} E_{k}\left(\omega^{i}(t)\right)\right)\right) / I
$$

where $E_{k}(\cdot)$ denotes the kth mode of EMD.

(6) Repeat steps (4) and (5), until all IMF are obtained.

\subsection{Extreme-point Symmetric Mode Decomposition (ESMD)}

ESMD is a new adaptive data processing method, which can be used to analysis non-stationary and nonlinear signals. ESMD uses internal extreme-point symmetry interpolation in place of external envelope interpolation, and optimize residual mode using the least square approach, which overcomes e shortcomings of modal aliasing and screening termination in EMD. The detailed steps of ESMD are as follows:

(1) Find all poles of series $Y$ and record them as $x_{i}(i=1,2, \cdots, n)$.

(2) Link adjacent $x_{i}$ with lines and mark the midpoints by $B_{i}(i=1,2, \cdots, n-$ 1).

(3) Add boundary midpoints $F_{0}$ and $F_{n}$ using direct interpolation.

(4) Construct $p$ bar differential curves, $L_{p}(p=1,2, \cdots, n)$, using the obtained midpoints, and compute the mean curve by $\bar{L}=\left(L_{1}+\cdots L_{p}\right) / p$.

(5) Repeat steps 1-4 on $Y-\bar{L}$ until $|\bar{L}| \leq \theta$ ( $\theta$ denotes the permitted error), and then the first mode $M_{1}$ is obtained.

(6) Repeat steps 1 to 5 on $\mathrm{Y}-M_{1}$ to obtain $M_{2}, \cdots, M_{n}$ and a residual (R) until 
$\mathrm{R}$ only has a certain number of poles.

(7) Adjust $\mathrm{K}$ within the interval $\left[K_{\min }, K_{\max }\right]$ and repeat steps 1 to 6 , then compute the standard variance $\sigma$ of $Y-R$.

(8) Select $K_{0}$ corresponding to a minimum $\sigma$, then $K_{0}$ is adopted to repeat steps 1 to 6 and output the final decomposition results.

After decomposition, the original series can generate a series of intrinsic mode functions (IMF) and one residual $(R)$.

\subsection{Singular Spectrum Analysis (SSA)}

SSA is a classical and powerful non-parametric signal preprocessing tool, which can identify and extract trends, noise and oscillatory behavior of time series (Zhang et al. 2017). The standard SSA contains four procedures, namely embedding, SVD (singular value decomposition), grouping and diagonal averaging. Embedding and SVD belong to data decomposition, and the latter two steps belong to data reconstruction. For further details, please refer to (Elsner 2002; Moreno et al. 2021; Wang et al. 2019), etc.

\subsection{Stepwise decomposition-based monthly runoff prediction technique}

The aim of this paper is to evaluate the potential of five data preprocessing techniques based on GRU model in monthly runoff series prediction. The methodological procedures of the study can be illustrated in Fig. 2. The modeling process is illustrated in the following steps:

(1) The original runoff data are decomposed into several subsequences using VMD, WPD, CEEMDAN, ESMD, and SSA. 
(2) For each extracted sub-sequence, GRU is adopted as a forecasting tool to model the split module, and to perform the corresponding forecasting for each sequence, respectively.

(3) The forecasting results of all components obtained by GRU are combined to generate the final forecasting results of different corresponding hybrid models.

\section{Insert Fig. 2.}

\section{Experimental analysis}

\subsection{Study area and data processing}

In this paper, two huge multipurpose reservoirs (Manwan and Hongjiadu) are taken as the research objects (Wang et al. 2009). Manwan Hydropower, located in the middle reach of Lancang River, is the first multimillion-kilowatt hydropower in Langcang River, with an installed capacity of $125 \times 10^{4} \mathrm{~kW}$. It is $132 \mathrm{~m}$ high, $148 \mathrm{~m}$ long and has $70 \mathrm{~km}$ backwater area. The drainage area controlled by the dam site is 114500 $\mathrm{km}^{2}$, with an average annual runoff of $1230 \mathrm{~m}^{3} / \mathrm{s}$. Hongjiadu reservoir is located in Wujiang River of Guizhou Province in China. Wujiang River is the largest tributary on the south bank of Yangtze River. Hongjiadu Hydropower is the leading reservoir of 11 cascade hydropower stations in Wujiang River with multi-year regulation capacity, which covers drainage area of $9900 \mathrm{~km}^{2}$, total reservoir storage of 4.95 billion $\mathrm{m}^{3}$, and the average annual runoff is $155 \mathrm{~m}^{3} / \mathrm{s}$.

Fig. 3 shows data for these two stations, where data for Manwan Hydropower runs from 1953 to 2004 and data for Hongjiadu station runs from 1951 to 2004. For Manwan Hydropower, data from 1953 to 1994 is adopted to train the model whilst that from 
2971994 is adopted to train the model whilst that from 1995 to 2004 is adopted for testing.

298 In order to avoid attributes within small range of values to be dominated by larger range 299 of values, and avoid numerical difficulties in the computation process, the normalization process is employed during modeling. As such, data of these two stations are normalized between $[0,1]$ by:

$$
x_{i}^{\prime}=\frac{x_{i}-\min _{1 \leq i \leq n}\left\{x_{i}\right\}}{\max _{1 \leq i \leq n}\left\{x_{i}\right\}-\min _{1 \leq i \leq n}\left\{x_{i}\right\}}
$$

\subsection{Evaluation indicators}

\section{Insert Fig. 3.}

Their equations are provided below.

$$
R M S E=\sqrt{\frac{1}{n} \sum_{i=1}^{n}\left(\mathrm{y}_{e}(i)-y_{o}(i)\right)^{2}}
$$

$$
M A P E=\frac{1}{N} \sum_{i=1}^{n}\left|\frac{y_{e}(i)-y_{o}(i)}{y_{e}(i)}\right| \times 100
$$

$$
\begin{gathered}
N S E C=1-\frac{\sum_{i=1}^{n}\left(y_{e}(i)-y_{o}(i)\right)^{2}}{\sum_{i=1}^{n}\left(y_{o}(i)-\overline{y_{o}}\right)^{2}} \\
R=\frac{\sum_{i=1}^{n}\left(\mathrm{y}_{o}(i)-\overline{y_{o}}\right)\left(y_{e}(i)-\overline{y_{e}}\right)}{\sqrt{\sum_{i=1}^{n}\left(y_{o}(i)-\overline{y_{o}}\right)^{2} \sum_{i=1}^{n}\left(y_{e}(i)-\overline{y_{e}}\right)^{2}}}
\end{gathered}
$$


where $y_{e}(i), y_{o}(i), \overline{y_{e}}$ and $\overline{y_{o}}$ are the estimated value, observed value, mean estimated value, and mean observed value of precipitation, respectively.

\subsection{Experimental results and discussion}

\subsubsection{Model development}

In this section, we investigate the performance of five data preprocessing techniques based on GRU model in monthly runoff series prediction, which are GUR, VMD-GRU, WPD-GRU, CEEMDAN-GRU, ESMD-GRU, and SSA-GRU. Detailed information and parameter settings relating to these models are presented as follows:

(1) GRU model

GRU is a variant of RNN, and the theory of GRU can be found in Section 2.1.

There are three significant parameters of GRU, namely the number of layers, hidden neurons, and iterations. In this study, these three parameters are set as 3, 16, and 100, respectively. The model is trained by Adam optimizer with 100 training epochs, the initial learning rate is adjusted to 0.001 , and MSE is adopted as the loss function. Note that the input variables have significant influence on the performance of GRU. However, there is no definite method to meet this practical necessity. Meanwhile, considering the number of sub-sequences and feature differences, the input variables are determined to be 12 by trial-and -error method.

(2) One-phase decomposition hybrid forecast models

For VMD-GRU, WPD-GRU, CEEMDAN-GRU, ESMD-GRU, and SSA-GRU models, the original data are decomposed into several subsequences using VMD, WPD, CEEMDAN ESMD, and SSA. Each subsequence is then modeled using GRU. The 
number of modes of VMD is determined as 8 . We adopt a three-scale and fourth order symlet wavelet as the wavelet basis function of WPD in this study. Before carrying out CEEMDAN on monthly runoff time series, values of trials and epsilon need to be determined in advance. After the trial-and -error procedure, they are set to be 100 and 0.2. For ESMD model, the number of iterations is 100 , and the number of remaining extreme points of Manwan and Hongjiadu are 8, 7 respectively. The number of window size and eigenvalue grouping of SSA are set to be 12 and 6 , respectively.

\subsubsection{Simulation results}

Tables 1 and 2 list the results of 6 forecasting models for Manwan and Hongjiadu reservoirs, respectively. One can see from Table 1 that when forecasting the monthly runoff in Manwan reservoir, VMD-GRU model consistently outperforms other models in terms of RMSE, MAPE, NSEC, and R in both training and testing phases. ESMDGRU model underperforms the naïve GRU model in Manwan reservoir. For example, in the training period, compared with GRU, ESMD-GRU, SSA-GRU, CEEMDANGRU, WPD-GRU, and VMD-GRU reduce RMSE by $-8.94 \%, 26.19 \%, 40.84 \%$, $72.82 \%$, and $80.32 \%$, respectively, reduce MAPE by $-73.75 \%, 3.13 \%,-12.05 \%$, $41.80 \%$, and $53.14 \%$, respectively, improve NSEC by $-3.86 \%, 9.40 \%, 13.43 \%, 19.12 \%$, and $19.86 \%$, respectively, improve R by $-2.06 \%, 4.41 \%, 6.44 \%, 8.98 \%$, and $9.32 \%$, respectively. In the testing phase, VMD-GRU attains the best RMSE, MAPE, NSEC, and R statistics of 78.980, 7.932, 0.993, and 0.997 , respectively. WPD-GRU performs slightly inferior to VMD-GRU in terms of these four predicting accuracy criteria. The analysis demonstrates that, except ESMD, the performance of the other four 
decomposition technologies combined with GUR are significantly better than that of the single GRU model, which means the effectiveness of decomposition technologies. Meanwhile, the performance of five data decomposition technologies developed in this study are different in terms of four numerical indicators. One can clearly see that the performance of VMD and WPD are significantly better than those of CEEMDAN, ESMD, and SSA methods, and VMD-GRU obtains the best results in terms of four indexes. Therefore, it can be concluded that extracting diversified fluctuation patterns from the original runoff time series by signal decomposition technologies significantly improves the forecasting accuracy of GRU model.

Table 2 presents the results obtained by six forecasting models for Hongjiadu reservoir. It can be seen that the results of GRU is inferior to those of GRU models with decompositions. This reaffirms the effectiveness of decomposition technologies. VMDGRU yields the best results in terms of four indexes. WPD-GRU performs slightly inferior to VMD-GRU in terms of four predicting accuracy criteria. Additionally, the performance of ESMD-GRU model is better than that of GRU in Hongjiadu reservoir. For example, in the testing phase, VMD-GRU obtain the best RMSE, MAPE, NSEC, and R statistics of $11.873,13.503,0.993$, and 0.997 , respectively. In the testing period, compared with GRU, WPD-GRU, reduce RMSE and MAPE by $85.96 \%$ and $63.72 \%$, improve NSEC and R by $53.00 \%$ and $22.18 \%$, respectively. The analysis again verifies the superior performance of VMD-GRU for monthly runoff forecasting.

Insert Table 1.

Insert Table 1. 

and Hongjiadu reservoirs, respectively. One can see from the scatter plots that all

381 forecasting models are capable of tracing changes in peak runoff to some extent, 382 demonstrating the effectiveness of the models. Observing the fitting line, we can see that the performance of VMD-GRU model in the two sites in both training and testing period are superior to the other five models, because its trend line is closer to the original data line and it can accurately track the change of peak runoff. Therefore, runoff in Manwan and Hongjiadu reservoirs.

\subsubsection{Discussion of the results}

The experimental results show that there are clear differences between VMD, WPD, CEEMDAN, ESMD, and SSA methods. Two conventional data preprocessing methods, namely ESMD and SSA, may suffer from some shortcomings, such as insufficient embedding dimension and weak generalization. SSA constructs the trajectory matrix according to empirical embedding dimension, which greatly affects the accuracy of data decomposition. According to the theory of ESMD, it is generally suitable for symmetrical data about the midpoint in each half cycle. For asymmetric data, the midpoint selected in ESMD is not representative. It is worth noting that 
CEEMDAN is significantly better than ESMD in capturing signal features. The reason may be that CEEMDAN reduces signal distortion and shift of characteristic peaks by decomposing Gaussian white noise using EMD, thus improving the accuracy. WPD decomposes both of previous detail coefficients and approximation, which offers richer signal analysis. In VMD, the center frequency and bandwidth of each variational model can be updated dynamically, which overcomes the end effect and mode mixing of EMD method. Meanwhile, VMD has a solid mathematical foundation, which can effectively reduce high complexity and nonlinearity of the sequence. The experimental results showed that VMD and WPD exhibit good performance for runoff time series forecasting. The analysis shown above appears to indicate that VMD-GRU and WPDGRU models can be implemented in actual monthly runoff forecasting.

Overall, the combination of data decomposition methods and forecasting models can obtain accurate monthly runoff prediction results for executors, which can provide valuable technical reference for hydrological forecasting research. It should be noted that there are still several research directions to be explored in future study. The first is to develop optimal computational parameters of GRU. Second, only two test cases are adopted in this paper, we need to test the generalization ability of the models. The last major aspect is that the research only considers historical runoff series, while other factors (such as rainfall and evaporation) could also be incorporated. In future research, it is necessary to explore researches based on the above three issues and develop more accurate prediction models to provide technical supports for hydrological time series forecasting. 


\section{Conclusions}

Decomposition-based hybrid methods are becoming increasingly popular in runoff time series forecasting, which decompose runoff data into several sub-series and forecast the sub-series separately. In this study, six forecasting models are developed for forecasting monthly runoff time series, namely GRU, ESMD-GRU, SSA-GRU, CEEMDAN-GRU, WPD-GRU, and VMD-GRU. The original monthly runoff series is first decomposed into several diversified subcomponents using five data preprocessing methods. Then, each component is predicted by constructing an appropriate GRU model. Manwan and Hongjiadu reservoirs are taken as the test cases. Four performance metrics are employed as the evaluation benchmarks. The experimental results indicate that VMD-GRU model outperforms several existing methods in terms of four statistical indexes. This demonstrates that VMD-GRU can significantly improve the prediction accuracy of monthly runoff time series. Meanwhile, the results also demonstrate that the signal feature extraction ability of VMD and WPD is significantly better than CEEMDAN, ESMD, and SSA methods. Therefore, it is significantly important to carefully determine an appropriate data preprocessing method according to actual characteristics of the study area, as well as deepen the research to study more accurate methods for hydrological time series forecasting.

Ethics Approval: All authors kept the 'Ethical Responsibilities of Authors'.

Consent to Participate: All authors gave explicit consent to participate in this work.

Consent to Publish: All authors gave explicit consent to publish this manuscript. 


\section{Authors' Contributions}

Wen-chuan Wang: Conceptualization, Methodology, Writing-original draft. Yu-jin

Du: Methodology, data curation, Writing - original draft preparation. Kwok-wing

Chau: Writing and editing-original draft. Chun-Tian Cheng: Formal analysis and data collection. Dong-mei Xu: Formal analysis. Wen-Tao Zhuang: Investigation.

\section{Funding}

Project of key science and technology of the Henan province (No: 202102310259; No: 202102310588), Henan province university scientific and technological innovation team (No: 18IRTSTHN009)

Competing of interest: The authors declare that they have no conflict of interest.

\section{Availability of data and materials}

All authors made sure that all data and materials support our published claims and comply with field standards.

\section{References}

Ali M, Prasad R, Xiang Y, Yaseen ZM (2020) Complete ensemble empirical mode decomposition hybridized with random forest and kernel ridge regression model for $\begin{array}{llll}\text { monthly rainfall forecasts Journal of Hydrology } 584 & \end{array}$ doi:10.1016/j.jhydrol.2020.124647

Bhandari S, Thakur B, Kalra A, Miller WP, Lakshmi V, Pathak P (2019) Streamflow Forecasting Using Singular Value Decomposition and Support Vector Machine for the Upper Rio Grande River Basin Journal of the American Water Resources Association 55:680-699 doi:10.1111/1752-1688.12733

Chen S, Dong SN, Cao ZG, Guo JT (2020a) A Compound Approach for Monthly Runoff Forecasting Based on Multiscale Analysis and Deep Network with Sequential Structure Water 12 doi:10.3390/w12082274

Chen X et al. (2020b) The importance of short lag-time in the runoff forecasting model based on long short-term memory Journal of Hydrology 589 doi:10.1016/j.jhydrol.2020.125359

Dragomiretskiy K, Zosso D (2014) Variational Mode Decomposition IEEE Transactions on Signal Processing 62:531-544 doi:10.1109/TSP.2013.2288675

Duan J et al. (2021) Short-term wind power forecasting using the hybrid model of 
improved variational mode decomposition and Correntropy Long Short -term memory neural network Energy 214:118980 doi:https://doi.org/10.1016/j.energy.2020.118980

Elsner JB (2002) Analysis of Time Series Structure: SSA and Related Techniques Journal of the American Statistical Association 97:1207-1208 doi:10.1198/jasa.2002.s239

Feng Z-k, Niu W-j, Tang Z-y, Jiang Z-q, Xu Y, Liu Y, Zhang H-r (2020) Monthly runoff time series prediction by variational mode decomposition and support vector machine based on quantum-behaved particle swarm optimization Journal of Hydrology 583 doi:10.1016/j.jhydrol.2020.124627

Fragkiadaki K, Levine S, Felsen P, Malik J Recurrent Network Models for Human Dynamics. In: 2015 IEEE International Conference on Computer Vision (ICCV), 7-13 Dec. 2015 2015. pp 4346-4354. doi:10.1109/ICCV.2015.494

Greff K, Srivastava RK, Koutnik J, Steunebrink BR, Schmidhuber J (2017) LSTM: A Search Space Odyssey IEEE Trans Neural Netw Learn Syst 28:2222-2232 doi:10.1109/tnnls.2016.2582924

He F, Zhou J, Feng Z-k, Liu G, Yang Y (2019) A hybrid short-term load forecasting model based on variational mode decomposition and long short-term memory networks considering relevant factors with Bayesian optimization algorithm Applied Energy 237:103-116 doi:https://doi.org/10.1016/j.apenergy.2019.01.055

Huang NE et al. (1998) The empirical mode decomposition and the Hilbert spectrum for nonlinear and non-stationary time series analysis Proceedings of the Royal Society of London Series A: Mathematical, Physical and Engineering Sciences 454:903-995 doi:10.1098/rspa.1998.0193

Kisi O, Cimen M (2011) A wavelet-support vector machine conjunction model for monthly streamflow forecasting Journal of Hydrology 399:132-140 doi:10.1016/j.jhydrol.2010.12.041

Kratzert F, Klotz D, Brenner C, Schulz K, Herrnegger M (2018) Rainfall-runoff modelling using Long Short-Term Memory (LSTM) networks Hydrology and Earth System Sciences 22:6005-6022 doi:10.5194/hess-22-6005-2018

LeCun Y, Bengio Y, Hinton G (2015) Deep learning Nature 521:436-444 doi:10.1038/nature14539

Liu H, Mi X, Li Y, Duan Z, Xu Y (2019) Smart wind speed deep learning based multi-step forecasting model using singular spectrum analysis, convolutional Gated Recurrent Unit network and Support Vector Regression Renewable Energy 143:842854 doi:10.1016/j.renene.2019.05.039

Liu H, Mi XW, Li YF (2018) Smart deep learning based wind speed prediction model using wavelet packet decomposition, convolutional neural network and convolutional long short term memory network Energy Conversion and Management 166:120-131 doi:10.1016/j.enconman.2018.04.021

Meshram SG, Singh VP, Kisi O, Karimi V, Meshram C (2020) Application of Artificial Neural Networks, Support Vector Machine and Multiple Model-ANN to Sediment Yield Prediction Water Resources Management 34:4561-4575 doi:10.1007/s11269-020-02672-8

Moreno SR, Mariani VC, Coelho LdS (2021) Hybrid multi-stage decomposition 
with parametric model applied to wind speed forecasting in Brazilian Northeast Renewable Energy 164:1508-1526 doi:10.1016/j.renene.2020.10.126

Niu W-j, Feng Z-k, Chen Y-b, Zhang H-r, Cheng C-t (2020) Annual Streamflow Time Series Prediction Using Extreme Learning Machine Based on Gravitational Search Algorithm and Variational Mode Decomposition Journal of Hydrologic Engineering 25 doi:10.1061/(asce)he.1943-5584.0001902

Ömer Faruk D (2010) A hybrid neural network and ARIMA model for water quality time series prediction Engineering Applications of Artificial Intelligence 23:586-594 doi:https://doi.org/10.1016/j.engappai.2009.09.015

Pan MY, Zhou HN, Cao JY, Liu YS, Hao JL, Li SX, Chen CH (2020) Water Level Prediction Model Based on GRU and CNN Ieee Access 8:60090-60100 doi:10.1109/access.2020.2982433

Ravanelli M, Brakel P, Omologo M, Bengio Y (2018) Light Gated Recurrent Units for Speech Recognition IEEE Transactions on Emerging Topics in Computational Intelligence 2:92-102 doi:10.1109/TETCI.2017.2762739

Reddy BSN, Pramada SK, Roshni T (2021) Monthly surface runoff prediction using artificial intelligence: A study from a tropical climate river basin Journal of Earth System Science 130 doi:10.1007/s12040-020-01508-8

Ren Y, Suganthan PN, Srikanth N (2015) A Comparative Study of Empirical Mode Decomposition-Based Short-Term Wind Speed Forecasting Methods IEEE Transactions on Sustainable Energy 6:236-244 doi:10.1109/TSTE.2014.2365580

Seo Y, Kim S, Kisi O, Singh VP, Parasuraman K (2016) River Stage Forecasting Using Wavelet Packet Decomposition and Machine Learning Models Water Resources Management 30:4011-4035 doi:10.1007/s11269-016-1409-4

Tang LH, Bai YL, Yang J, Lu YN (2020) A hybrid prediction method based on empirical mode decomposition and multiple model fusion for chaotic time series Chaos Solitons \& Fractals 141 doi:10.1016/j.chaos.2020.110366

Turner SWD, Doering K, Voisin N (2020) Data-Driven Reservoir Simulation in a Large-Scale Hydrological and Water Resource Model Water Resources Research 56 doi:10.1029/2020wr027902

Wang B, Wang J (2021) Energy futures price prediction and evaluation model with deep bidirectional gated recurrent unit neural network and RIF-based algorithm Energy 216 doi:10.1016/j.energy.2020.119299

Wang L, Li X, Ma C, Bai Y (2019) Improving the prediction accuracy of monthly streamflow using a data-driven model based on a double-processing strategy Journal of Hydrology 573:733-745 doi:10.1016/j.jhydrol.2019.03.101

Wang W-C, Chau K-W, Cheng C-T, Qiu L (2009) A comparison of performance of several artificial intelligence methods for forecasting monthly discharge time series Journal of Hydrology 374:294-306 doi:https://doi.org/10.1016/j.jhydrol.2009.06.019

Wang W-c, Chau K-w, Qiu L, Chen Y-b (2015a) Improving forecasting accuracy of medium and long-term runoff using artificial neural network based on EEMD decomposition Environmental Research 139:46-54 doi:10.1016/j.envres.2015.02.002

Wang W-c, Chau K-w, Xu D-m, Chen X-Y (2015b) Improving Forecasting Accuracy of Annual Runoff Time Series Using ARIMA Based on EEMD 
Decomposition Water Resources Management 29:2655-2675 doi:10.1007/s11269-0150962-6

Wang W-c, Xu D-m, Chau K-w, Chen S (2013) Improved annual rainfall-runoff forecasting using PSO-SVM model based on EEMD Journal of Hydroinformatics 15:1377-1390 doi:10.2166/hydro.2013.134

Xiang Z, Yan J, Demir I (2020) A Rainfall-Runoff Model With LSTM-Based Sequence-to-Sequence Learning Water Resources Research 56 doi:10.1029/2019wr025326

Yaseen ZM, Jaafar O, Deo RC, Kisi O, Adamowski J, Quilty J, El-Shafie A (2016) Stream-flow forecasting using extreme learning machines: A case study in a semi-arid region in Iraq Journal of Hydrology 542:603-614 doi:10.1016/j.jhydrol.2016.09.035

Yaseen ZM et al. (2019) Implementation of Univariate Paradigm for Streamflow Simulation Using Hybrid Data-Driven Model: Case Study in Tropical Region Ieee Access 7:74471-74481 doi:10.1109/access.2019.2920916

Yu CJ, Li YL, Bao YL, Tang HJ, Zhai GH (2018) A novel framework for wind speed prediction based on recurrent neural networks and support vector machine Energy Conversion and Management 178:137-145 doi:10.1016/j.enconman.2018.10.008

Yu JX, Zhang X, Xu LL, Dong J, Zhangzhong LL (2021) A hybrid CNN-GRU model for predicting soil moisture in maize root zone Agricultural Water Management 245 doi:10.1016/j.agwat.2020.106649

Yue ZX, Ai P, Xiong CS, Hong M, Song YH (2020) Mid- to long-term runoff prediction by combining the deep belief network and partial least-squares regression Journal of Hydroinformatics 22:1283-1305 doi:10.2166/hydro.2020.022

Zhang GW, Liu D (2020) Causal convolutional gated recurrent unit network with multiple decomposition methods for short-term wind speed forecasting Energy Conversion and Management 226 doi:10.1016/j.enconman.2020.113500

Zhang Q, Wang B-D, He B, Peng Y, Ren M-L (2011) Singular Spectrum Analysis and ARIMA Hybrid Model for Annual Runoff Forecasting Water Resources Management 25:2683-2703 doi:10.1007/s11269-011-9833-y

Zhang X, Wang J, Zhang K (2017) Short-term electric load forecasting based on singular spectrum analysis and support vector machine optimized by Cuckoo search algorithm Electric Power Systems Research 146:270-285 doi:https://doi.org/10.1016/j.epsr.2017.01.035

Zhao XH, Lv HF, Wei YZ, Lv SJ, Zhu XP (2021) Streamflow Forecasting via Two Types of Predictive Structure-Based Gated Recurrent Unit Models Water 13 doi:10.3390/w13010091

Zuo GG, Luo JG, Wang N, Lian YN, He XX (2020) Two-stage variational mode decomposition and support vector regression for streamflow forecasting Hydrology and Earth System Sciences 24:5491-5518 doi:10.5194/hess-24-5491-2020 
605 Table captions

606 Table 1. Composition of different models for Manwan reservoir.

607 Table 2. Composition of different models for Hongjiadu reservoir. 
609

610

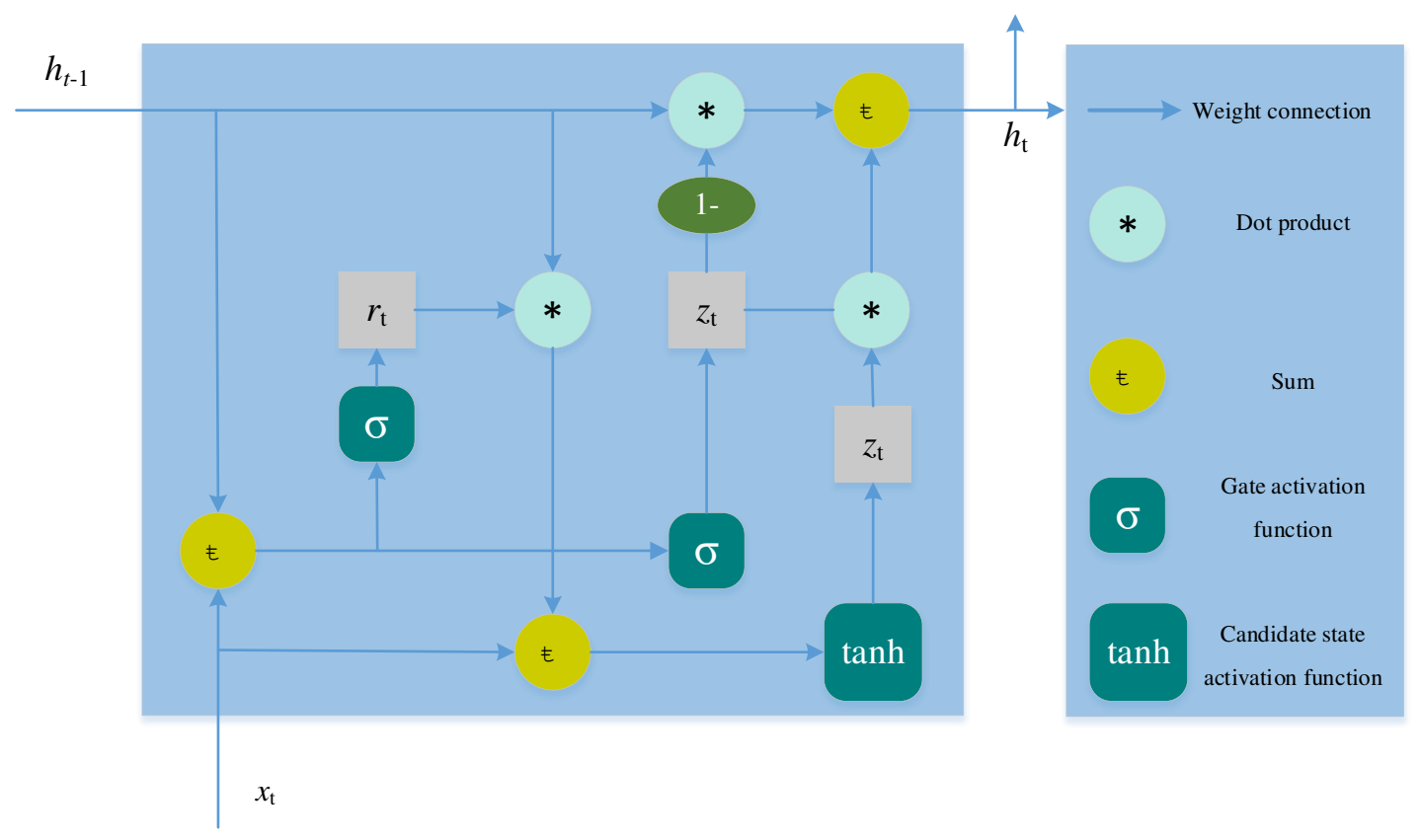

Fig. 1. Architecture of GRU

611

612

613

614

615

616

617

618

619

620

621 


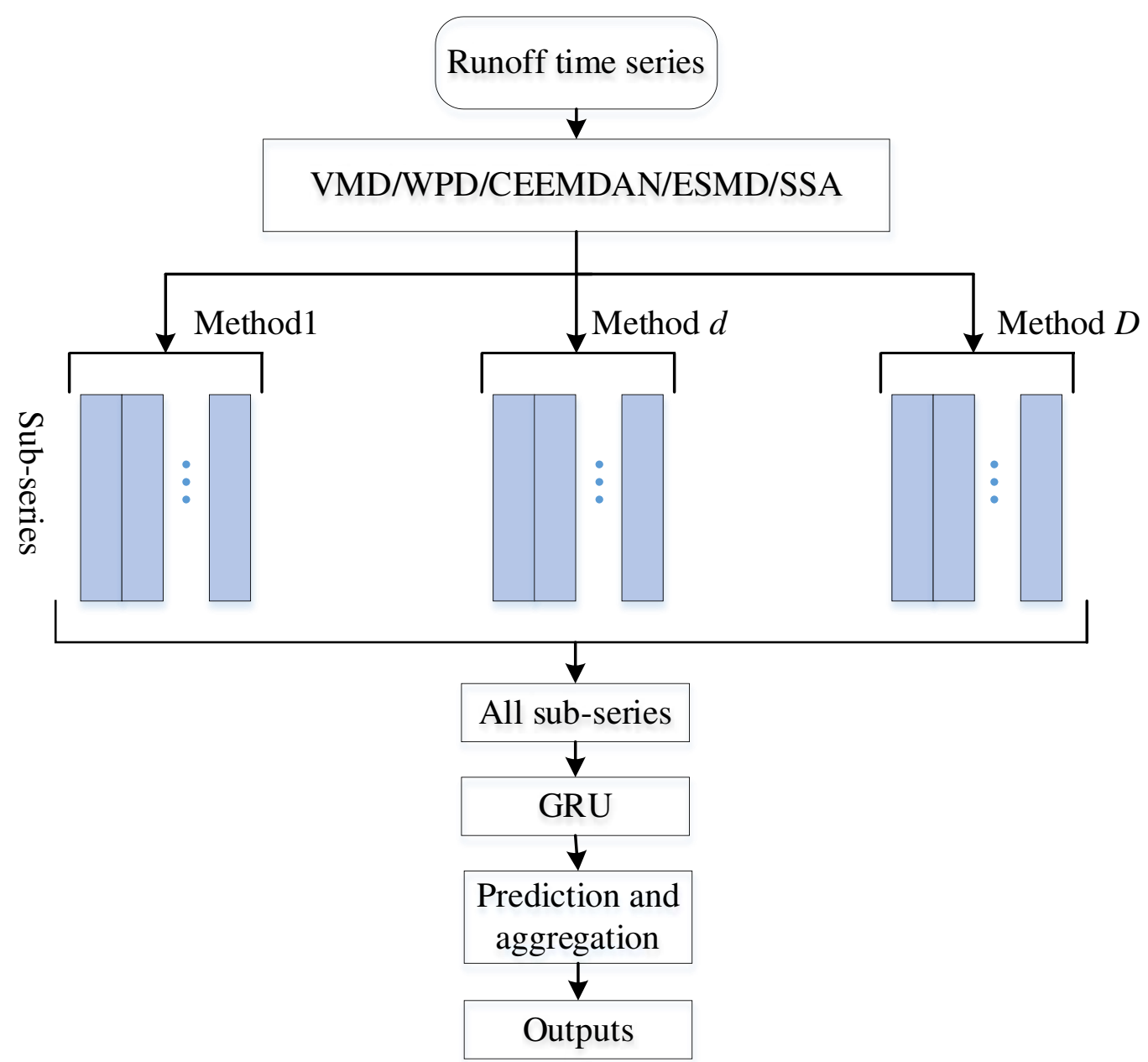

Fig. 2. The methodological procedures of the study

623

624

625

626

627

628

629

630 


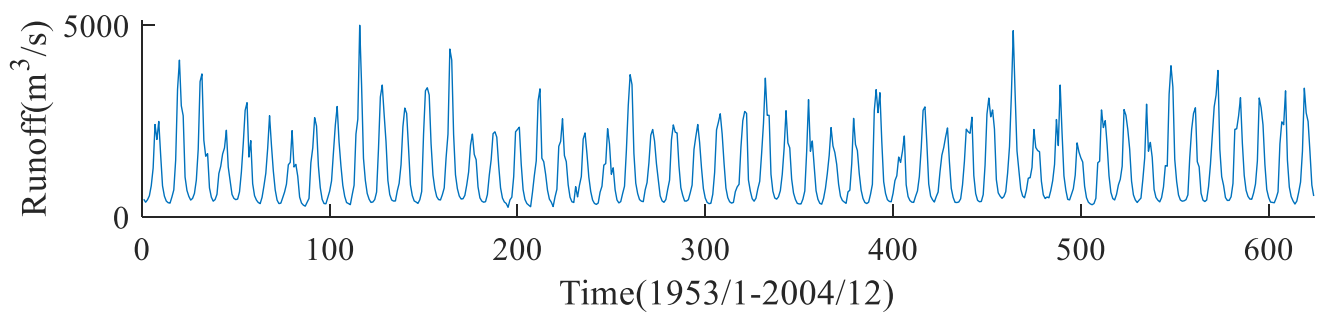

(a)Manwan reservoir

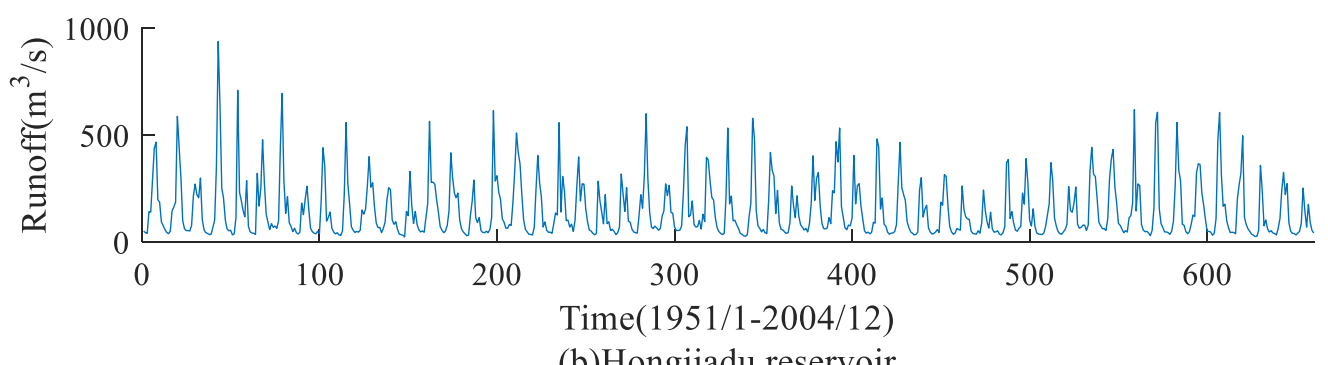

631

632

633

634

635

636

637 638

Fig. 3. Monthly runoff data for Manwan and Hongjiadu reservoirs.

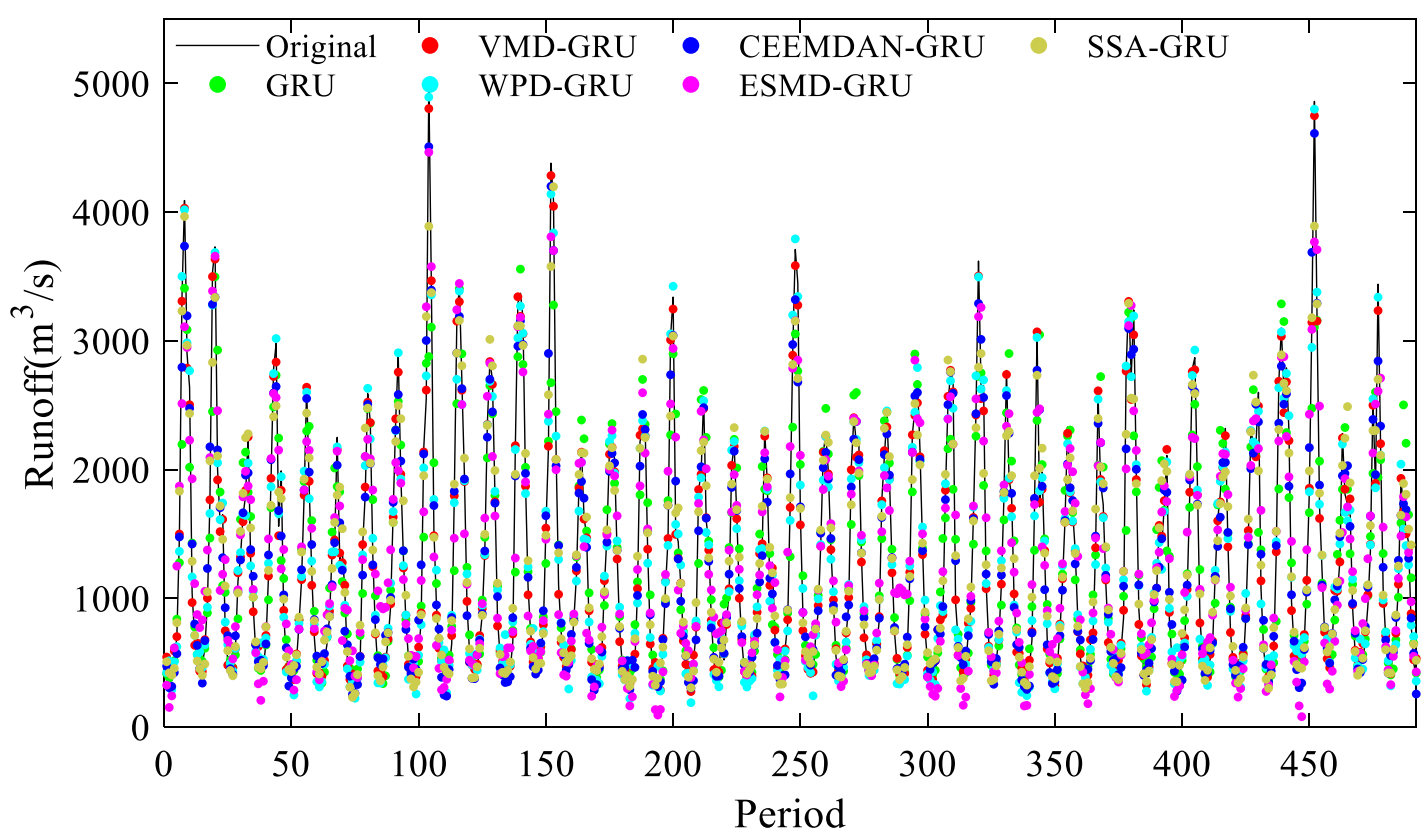

Fig. 4. Forecasting results of six models for Manwan reservoir during the training period. 


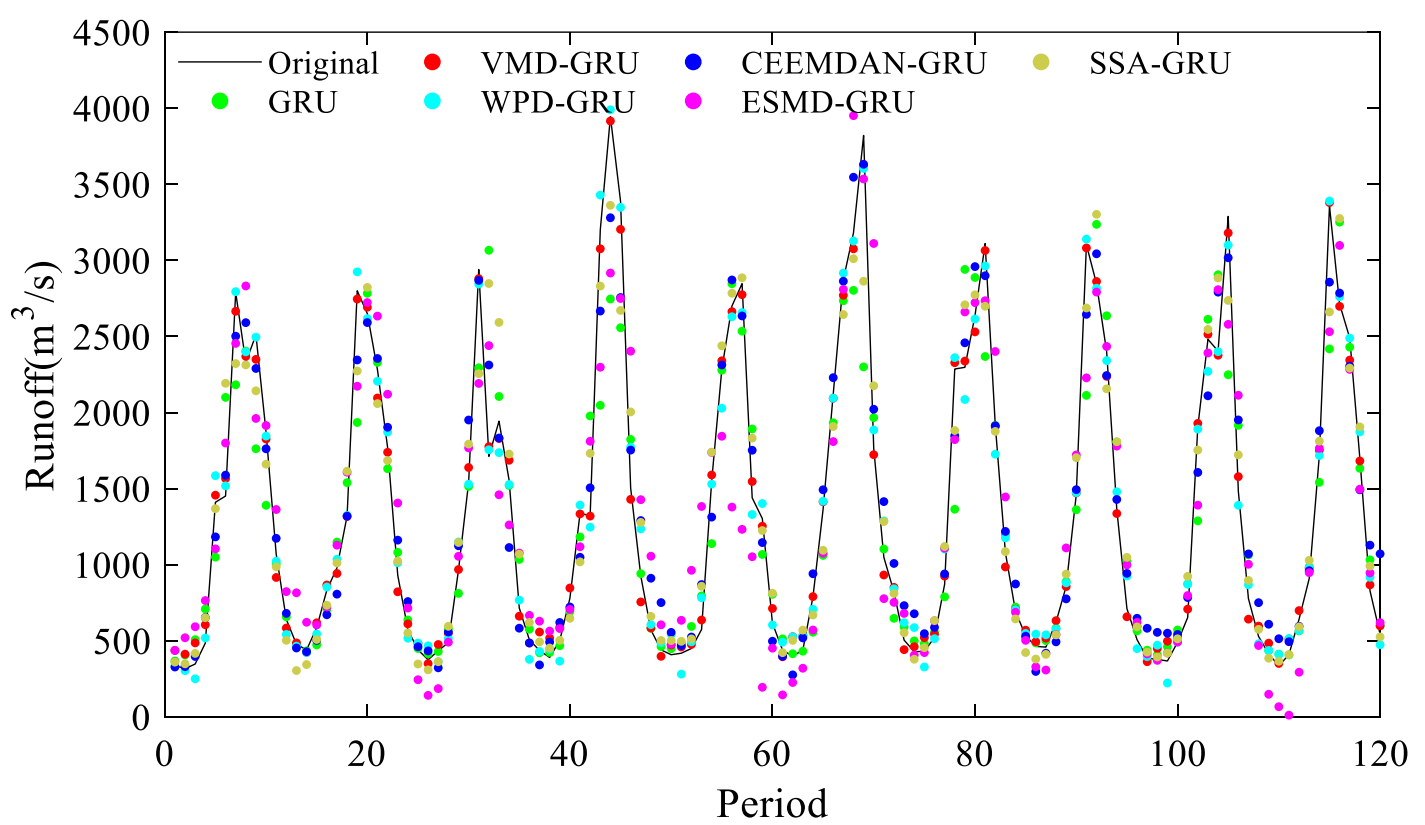

640 Fig. 5. Forecasting results of six models for Manwan reservoir during the testing period.

641

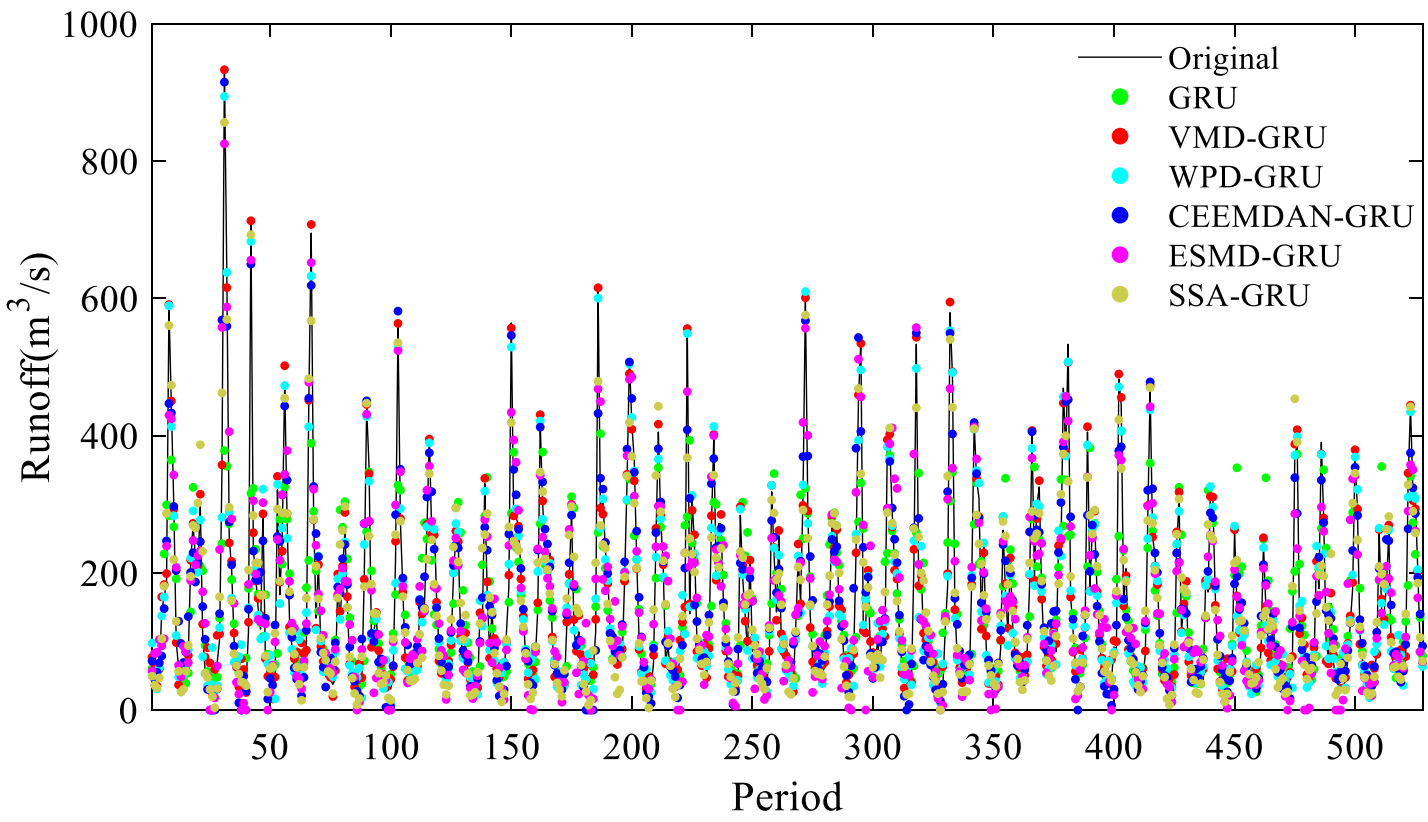

642

643 Fig. 6. Forecasting results of six models for Hongjiadu reservoir during the training 644 period. 


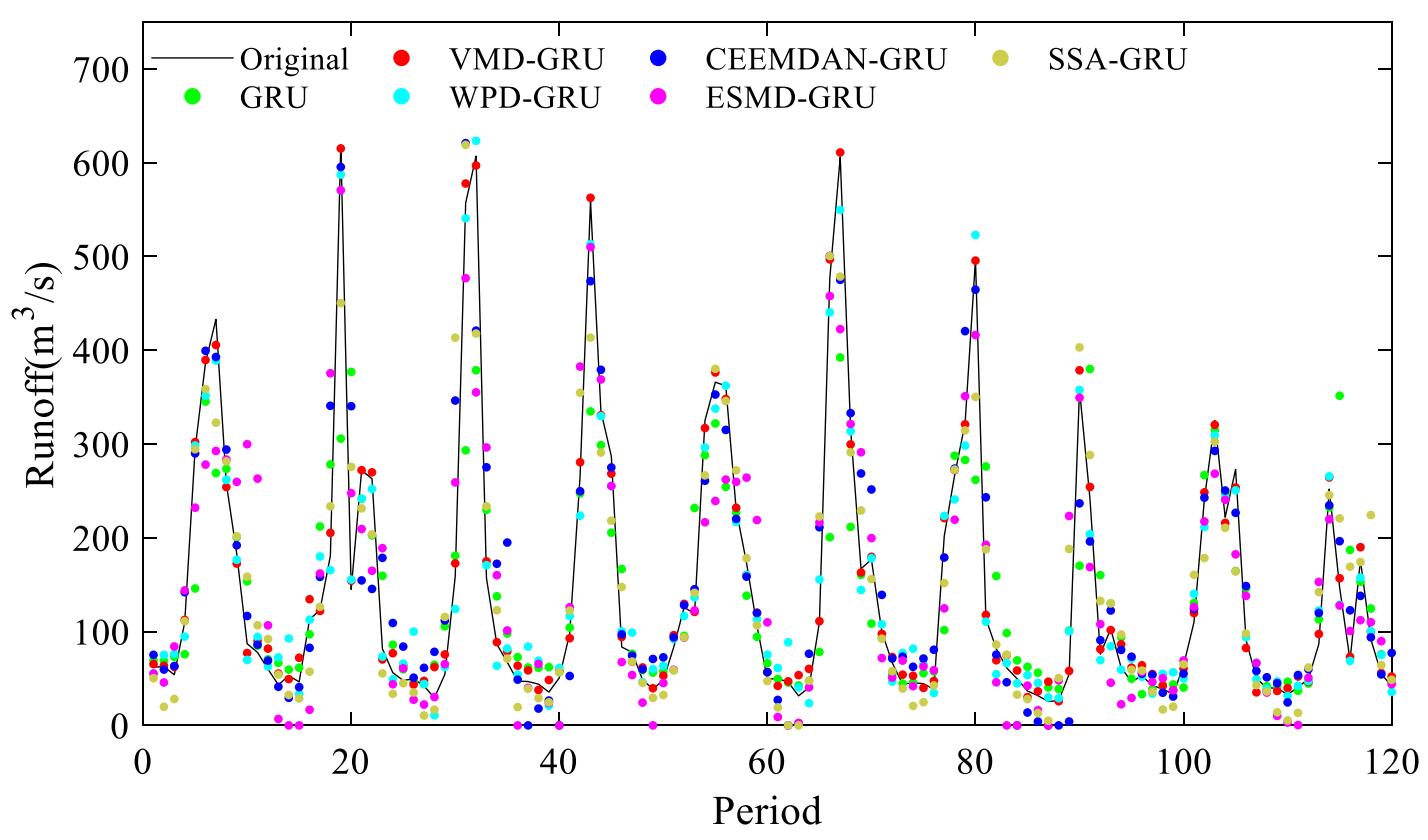

646 Fig. 7. Forecasting results of six models for Hongjiadu reservoir during the testing 647 period.

648 649 Table 1 Composition of different models for Manwan reservoir.

\begin{tabular}{ccccccccc}
\hline \multirow{2}{*}{ Model } & Training & \multicolumn{7}{c}{ Testing } \\
& RMSE & MAPE & NSEC & R & RMSE & MAPE & NSEC & R \\
\hline GRU & 343.665 & 16.786 & 0.857 & 0.927 & 401.336 & 16.925 & 0.829 & 0.912 \\
ESMD-GRU & 306.810 & 26.260 & 0.886 & 0.942 & 437.218 & 29.406 & 0.797 & 0.894 \\
SSA-GRU & 213.358 & 13.250 & 0.945 & 0.974 & 296.236 & 16.395 & 0.907 & 0.953 \\
CEEMDAN-GRU & 168.336 & 12.367 & 0.966 & 0.984 & 237.417 & 18.964 & 0.940 & 0.971 \\
WPD-GRU & 105.276 & 9.952 & 0.987 & 0.994 & 109.095 & 9.850 & 0.987 & 0.994 \\
VMD-GRU & 64.639 & 6.878 & 0.995 & 0.998 & 78.980 & 7.932 & 0.993 & 0.997 \\
\hline
\end{tabular}

650

651 Table 2 Composition of different models for Hongjiadu reservoir.

\begin{tabular}{ccccccccc}
\hline \multirow{2}{*}{ Model } & Training & \multicolumn{7}{c}{ Testing } \\
& RMSE & MAPE & NSEC & R & RMSE & MAPE & NSEC & R \\
\hline GRU & 84.022 & 38.232 & 0.597 & 0.774 & 84.561 & 37.225 & 0.649 & 0.816 \\
ESMD-GRU & 56.710 & 45.040 & 0.817 & 0.906 & 71.519 & 51.569 & 0.749 & 0.868 \\
SSA-GRU & 45.709 & 29.216 & 0.881 & 0.939 & 58.226 & 36.707 & 0.834 & 0.913 \\
CEEMDAN-GRU & 43.396 & 31.255 & 0.893 & 0.946 & 57.627 & 41.130 & 0.837 & 0.918 \\
WPD-GRU & 19.098 & 17.292 & 0.979 & 0.990 & 22.188 & 21.276 & 0.976 & 0.990 \\
VMD-GRU & 12.580 & 12.618 & 0.991 & 0.996 & 11.873 & 13.503 & 0.993 & 0.997 \\
\hline
\end{tabular}

